\title{
Cytotoxicity of local anesthetics on rabbit adipose-derived mesenchymal stem cells during early chondrogenic differentiation
}

\author{
TAO WU ${ }^{1}$, ZHAOHONG SHI ${ }^{2}$, HAIXIN SONG ${ }^{1}$, YANGZHENG LI ${ }^{1}$ and JIAN-HUA LI ${ }^{1}$ \\ ${ }^{1}$ Department of Rehabilitation Medicine, Sir Run Run Shaw Hospital, Zhejiang University School of Medicine, \\ Hangzhou, Zhejiang 310016; ${ }^{2}$ Department of Rehabilitation Medicine, The First People's Hospital of Wenling, Wenling, \\ Zhejiang 317500, P.R. China
}

Received February 3, 2018; Accepted June 1, 2018

DOI: $10.3892 /$ etm.2018.6539

\begin{abstract}
Local anesthetics (LAs) are commonly used to provide peri-operative pain control in the peripheral joints. In the field of regenerative medicine, adipose-derived mesenchymal stem cells (ADMSCs) are gaining attention as a cellular source for repair and regeneration in degenerative diseases. However, previous studies have demonstrated that the commonly used drugs lidocaine, ropivacaine, bupivacaine and mepivacaine may be toxic to human chondrocytes, which has raised concerns over whether they exert similar negative effects on ADMSCs during early chondrogenic differentiation. In the present in vitro study, the cytotoxicity of different LAs to ADMSCs was determined during early chondrogenic differentiation. At concentrations similar to those after physiological dilution once injected into the degenerative tissues, LAs ( $1 \%$ lidocaine, $0.5 \%$ bupivacaine, $0.5 \%$ ropivacaine or $2 \%$ mepivacaine) and PBS (control group) were incubated with rabbit ADMSCs (rADMSCs) for $60 \mathrm{~min}$. Following further culture for 3 or 7 days, the cell viability, apoptosis and morphological alterations of chondrogenic differentiation were measured by determining the mitochondrial activity, by flow cytometric analysis, Safranine Fast Green double staining and reverse transcription-quantitative polymerase chain reaction of chondrogenesis-associated genes. The results indicated that the mitochondrial activity in rADMSC was decreased and the apoptotic rate was increased, following treatment with LAs $(\mathrm{P}<0.05)$. Lidocaine $(1 \%)$ was less cytotoxic to rADMSCs during early chondrogenesis compared with other LAs. The expression levels of chondrogenesis-associated markers, including collagen I, collagen III and sex-determining region $\mathrm{Y}$ box 9 were all decreased at day 3 following exposure to
\end{abstract}

Correspondence to: Dr Jian-Hua Li, Department of Rehabilitation Medicine, Sir Run Run Shaw Hospital, Zhejiang University School of Medicine, 3 East Qin Chun Road, Hangzhou, Zhejiang 310016, P.R. China

E-mail: jianhua_li@zju.edu.cn

Key words: mesenchymal stem cells, local anesthetics, cytotoxicity, chondrogenic differentiation
LAs compared with the control group $(\mathrm{P}<0.05)$. The expression levels of these chondrogenesis-associated genes began to increase on day 7 following exposure but remained lower compared with the control group $(\mathrm{P}<0.05)$. Of note, $2 \%$ mepivacaine and $1 \%$ lidocaine exhibited a less pronounced negative effect on chondrogenesis-associated gene expression compared with other LAs. Therefore, the present study concluded that LAs are cytotoxic to rADMSCs during early chondrogenesis. Attention should be paid to the different types of LA selected in conjunction with ADMSC injection therapy.

\section{Introduction}

Degenerative musculoskeletal and spine conditions are significant causes of pain and disability in patients worldwide (1). In recent decades, advances in regenerative medicine and cell-based therapies, particularly the transplantation of mesenchymal stem cells (MSCs), have led to numerous studies and clinical trials utilizing these biological therapies to treat degenerative conditions, frequently reporting favorable outcomes (2). The increase in the number of therapeutic MSC procedures in the peripheral joint and in spine interventions is causing a growing concern regarding the potential toxicity of co-injectable drugs to MSCs.

Intra-articular administration of amide-type local anesthetics (LAs) is routinely performed during arthroscopic joint surgery (3). In orthopedic cartilage repair operations, the delivery of human MSCs is often required via intra-articular injection and it is common to introduce LAs prior to, during and following this procedure (4). However, a number of in vitro experimental studies using the same concentrations of LAs as in the clinical setting suggested its cytotoxic effect on articular chondrocytes (5) and human MSCs (6-8). While multiple in vitro trials have been performed, they were frequently limited by small sample size, heterogeneity in study design and conflicting outcomes, and therefore, the impact of LAs on adipose-derived mesenchymal stem cells (ADMSCs) remains to be elucidated.

To the best of our knowledge, the response of ADMSCs to LAs during chondrogenic differentiation has remained largely elusive. Breu et al (9) demonstrated that LAs have cytotoxic effects on bone marrow-derived MSCs (BMSCs) undergoing chondrogenesis, particularly in the superficial 
layers. Bupivacaine, ropivacaine and mepivacaine did not differ in cytotoxicity on MSC in aggregates. To the best of our knowledge, the present study was the first to report the cytotoxicity of LAs to human MSCs during chondrogenesis. The study by Breu et al had several limitations. First, only three types of LA were used and lidocaine was not included. In addition, the BMSCs were exposed to LAs at 7 days after chondrogenesis, which is not an ideal time-point to replicate clinical conditions, as MSCs are always injected following LA-induced anesthesia. Finally, the evaluation index for cell viability and response to LA exposure during chondrogenesis was limited as cell viability, apoptosis and necrosis were only evaluated after LA exposure.

Therefore, the present in vitro study was designed to determine the cytotoxic effect of different types of LA on rabbit ADMSCs (rADMSCs) during early chondrogenesis. The present study aimed to identify a safe type and concentration of LA for ADMSCs. The results of the present study may assist clinicians in selecting the optimal LA concentration during regenerative procedures and serve as a foundation for future research examining the cytotoxicity of LAs to ADMSCs during early chondrogenic differentiation.

\section{Materials and methods}

General design. rADMSCs were exposed to LA: 1\% lidocaine, $0.5 \%$ bupivacaine (both Beijing Solarbio Science \& Technology Co., Ltd., Beijing, China), 0.5\% ropivacaine (AuroMedics Pharma LLC Pharma LLC., East Windsor, NJ, USA) or $2 \%$ mepivacaine (Hospira Inc., Lake Forest, IL, USA) for $60 \mathrm{~min}$. The control groups were treated with complete PBS for $60 \mathrm{~min}$. Following the incubations, the cells were removed from the solutions and incubated in culture medium [Dulbecco's modified Eagle's medium supplemented with 10\% fetal bovine serum (Hyclone; GE Healthcare, Little Chalfont, UK)] Analyses of cell viability, apoptosis, morphological alterations and chondrogenesis-associated gene expression were performed early after exposure (day 3) and later (day 7).

Cell isolation, culture conditions and in vitro chondrogenic differentiation. A total of 5 healthy New Zealand rabbits weighing 2.5-3.0 kg and aged 2-3 months were provided by the Experimental Animal Centre of Zhejiang University Affiliated with Sir Run Run Shaw Hospital (SRRSH; Hangzhou, China). The experimental protocol was approved by the Animal Ethics Committee of Zhejiang University Affiliated with SRRSH Hospital (Hangzhou, China).

Primary rADMSCs were harvested from the adipose tissue in the groin of the New Zealand rabbits as previously described (10). Adipose tissue was digested with Type I collagenase (Sigma-Aldrich; Merck KGaA, Darmstadt, Germany) for $1.5 \mathrm{~h}$ at $37^{\circ} \mathrm{C}$, centrifuged at $400 \mathrm{x}$ g for $5 \mathrm{~min}$, rinsed with PBS and passed through $100-\mu \mathrm{m}$ cell strainers (Shanghai Bolting Cloth Manufacturing Co., Ltd., Shanghai, China). The remaining rADMSCs (those which passed through the strainer) were cultured in culture medium. The culture medium was replaced every 3 days and non-adherent cells were removed. In the present study, adherent primary rADMSCs exhibited a fibroblastoid and typical spindle-shaped morphology. The presence of CD29 and CD44 was detected by flow cytometry to confirm the identity of the rADMSCs (data not shown). All rADMSCs used in the subsequent experiments were of passage 7 .

The cells were cultured in $175 \mathrm{ml}$ flasks at $37^{\circ} \mathrm{C}$ in a humidified atmosphere containing $5 \% \mathrm{CO}_{2}$ until $90 \%$ confluence. Subsequently, they were trypsinized (TrypLE Express; Thermo Fisher Scientific, Inc., Waltham, MA, USA) and seeded onto 96-well plates (for MTS analysis), and onto 6-well plates [for flow cytometry, Safranine Fast Green double staining and reverse transcription-quantitative polymerase chain reaction (RT-qPCR) analysis] at a density of 10,000 cells $/ \mathrm{cm}^{2}$. The rADMSCs were maintained in chondrogenic differentiation media which consisted of culture media supplemented with $6.25 \mu \mathrm{g} / \mathrm{ml}$ insulin (Shanghai Orgchem Co., Ltd., Shanghai, China), $6.25 \mu \mathrm{g} / \mathrm{ml}$ transferrin (Shanghai Orgchem Co., Ltd.), $50 \mu \mathrm{g} / \mathrm{ml}$ ascorbic acid (Sigma-Aldrich; Merck KGaA), $10 \mathrm{ng} / \mathrm{ml}$ transforming growth factor (TGF)- $\beta 1$ (Peprotech, Inc., Rocky Hill, NJ, USA) and $0.1 \mu \mathrm{mol} / 1$ dexamethasone (Sigma-Aldrich; Merck KGaA). To evaluate the response of rADMSCs to exposure to LAs, all cells were allowed to differentiate for up to 3 days in chondrogenic differentiation media prior to LA treatment.

Treatment groups. rADMSCs were exposed to $1 \%$ lidocaine, $0.5 \%$ bupivacaine, $0.5 \%$ ropivacaine, $2 \%$ mepivacaine or complete PBS as a control. rADMSC cultures were subdivided into triplicate wells per condition and exposed to LAs for $60 \mathrm{~min}$. Following exposure, the cells were washed with $1 \mathrm{X}$ PBS and returned to the incubator in fresh chondrogenesis culture media. rADMSC viability was measured on days 3 and 7 post-exposure.

Assessment of ADMSC viability. To assess the metabolic activity of ADMSCs post-LA exposure, MTS colorimetric assays (Promega Corp., Madison, WI, USA) were performed at days 3 and 7 post-exposure. MTS assays (11) were evaluated using a Spectra Max plus Plate Reader (Molecular Devices, Sunnyvale, CA, USA) at a wavelength of $490 \mathrm{~nm}$. The assay is based on the reduction of the levels of the MTS tetrazolium compound by viable cells to generate a colored formazan product that is soluble in cell culture media. This conversion is performed by NADPH-dependent dehydrogenase enzymes in metabolically active cells. The formazan dye produced by viable cells may be quantified by measuring the absorbance at $490 \mathrm{~nm}$.

Analysis of rADMSC apoptosis. Cell apoptosis was measured by flow cytometry. An Annexin V-FITC Apoptosis Detection kit (Accuri C6; BD Biosciences, Franklin Lakes, NJ, USA) was used to evaluate the percentage of apoptotic cells. rADMSCs were treated with trypsin (without EDTA), washed two times with PBS and centrifuged $\left(750 \mathrm{x} \mathrm{g}, 5 \mathrm{~min}, 37^{\circ} \mathrm{C}\right)$ to collect the pellets. Subsequently, $5 \times 10^{4}$ treated cells were re-suspended in $300 \mu \mathrm{l}$ cold $1 \mathrm{X}$ binding buffer, after which $5 \mu \mathrm{l}$ Annexin $\mathrm{V}$-FITC and $10 \mu \mathrm{l}$ propidium iodide were added and the suspension was analyzed by flow cytometry (FACScan; BD Biosciences).

Morphological alterations during chondrogenic differentiation. Cell morphological alterations during chondrogenic 
differentiation were measured by Safranine Fast Green double staining. A total of $8 \times 10^{4}$ cells were seeded into each well of a 6-well plate containing microscopic slides and cultured to $80 \%$ confluency at $37^{\circ} \mathrm{C}$ with $5 \% \mathrm{CO}_{2}$. Following culture, the cells were rinsed with PBS three times for $1 \mathrm{~min}$ each and fixed for $2 \mathrm{~h}$ in cold paraformaldehyde solution. The slides were placed in histology cassettes and stored in $70 \%$ ethanol. Subsequently, $1 \%$ safranin (Solarbio, Beijing, China) and $0.02 \%$ Fast Green (CAS no. 2353-45-9; Jianglai Bio, Shanghai, China) double staining was performed (12). Finally, after dewaxing with xylene and sealing with neutral glycerol, the slides were observed under a light microscope (Nikon, Tokyo, Japan).

Genetic analysis of the response of rADMSCs to exposure to $L A s$. To determine the effect of LA exposure on rADMSCs during chondrogenic differentiation for clinical applications in cartilage regeneration, rADMSCs were subjected to RT-qPCR analysis of chondrogenic-associated gene expression. Prior to RT-qPCR, RNA was isolated and reverse transcribed using the RT-qPCR kit (TransGen Biotech, Beijing, China) Subsequently, the complementary DNA obtained was amplified by RT-qPCR (CFX384 Real-Time System; Bio-Rad Laboratories, Inc., Hercules, CA, USA) with SYBR Green detection using primers specific to chondrogenesis-associated genes, including collagen I, collagen III and sex-determining region Y box (SOX)9, whose sequences are listed in Table I. Gene expression levels were quantified using the $2^{-\Delta \Delta \mathrm{Cq}}$ method (13). The mRNA expression of the target genes was normalized to the housekeeping gene GAPDH. All of the samples were assayed in triplicate.

Statistical analysis. Descriptive statistics were utilized to demonstrate the effects of LA exposure on rADMSC viability (mitochondrial activity, MTS results), the apoptotic rate and the mRNA expression on days 3 and 7 post-exposure. The results were analyzed by one-way analysis of variance followed by a least-significant differences post-hoc test using GraphPad Prism software (version 6.0; GraphPad Software, Inc., La Jolla, CA, USA). $\mathrm{P}<0.05$ was considered to indicate a statistically significant difference. Values are expressed as the mean \pm standard deviation.

\section{Results}

Effect of LAs on rADMSC viability. The viability of rADMSC based on their mitochondrial activity was assessed by an MTS assay. It was determined that the mitochondrial activity was decreased at 3 days following treatment with LAs compared with that in the PBS control $(\mathrm{P}<0.05$; Fig. 1). Treatment with $1 \%$ lidocaine resulted in reduced toxic effects on mitochondrial activity compared with the other LAs $(\mathrm{P}<0.05)$. Furthermore, mitochondrial activity of rADMSCs recovered to a certain extent at day 7 in all LA exposure groups, but remained decreased in all LA treatment groups compared with the PBS control $(\mathrm{P}<0.05)$. On day 7 , the mitochondrial activity in the $1 \%$ lidocaine- and 2\% mepivacaine-treated groups was higher than that in the groups treated with $0.5 \%$ ropivacaine or $0.5 \%$ bupivacaine. Cells treated with $0.5 \%$ bupivacaine had markedly lower mitochondrial activity compared with the other 3 LAs. Therefore, the recovery abilities of the cells treated with $1 \%$ lidocaine and $2 \%$ mepivacaine were improved when compared with those in the other two LA groups $(\mathrm{P}<0.05)$.

Effect of LAs on rADMSC apoptosis. The apoptotic rate of rADMSCs was measured by flow cytometry. It was determined that the apoptotic rate increased on days 3 and 7 after treatment with LAs compared with that in the PBS-treated control ( $\mathrm{P}<0.05$; Fig. 2). The apoptotic rate in the $1 \%$ lidocaine and $0.5 \%$ ropivacaine groups was lower compared with that in the groups treated with $2 \%$ mepivacaine- and $0.5 \%$ bupivacaine groups (on day 7). Therefore, treatment with $1 \%$ lidocaine or $0.5 \%$ ropivacaine resulted in a lower apoptotic rate compared with that in the groups treated with the other LAs ( $\mathrm{P}<0.05$; Fig. 2).

Effect of LAs on morphological alterations of chondrogenic differentiation. rADMSCs cultured in chondrogenic medium formed aggregates and exhibited typical pink staining for glycosaminoglycans and morphological features, including enlarged lacunae around cells, typical of cartilage (safranine Fast Green double staining). In all LA treatment groups, less mature cartilage compared with that in the PBS group was identified using the staining on days 3 and 7 after the $60-$ min exposure (Fig. 3). By contrast, the aggregates in the $1 \%$ lidocaine treatment group resembled more mature cartilage compared with that in the group treated with the other LAs, as demonstrated by the staining (Fig. 3).

Effect of LAs on chondrogenesis-associated mRNA expression. The effect of a 60-min LA exposure on chondrogenesis-associated mRNA expression was evaluated on days 3 and 7. As expected, chondrogenesis-associated markers, including collagen I, collagen III and SOX9, were all decreased at day 3 after LA exposure compared with those in the control group ( $\mathrm{P}<0.05$; Fig. 4). In comparison with day 3 , these chondrogenesis-associated genes began to increase in all groups on day 7 after exposure but remained lower compared with those in the control group $(\mathrm{P}<0.05$; Fig. 4). Exposure to LAs decreased the chondrogenic ability of rADMSCs. The mRNA expression levels of collagen III and SOX9 were higher in the lidocaine group and, collagen I, collagen III and SOX9 were higher in the mepivacaine group compared with that in the other LA-treated groups. These results demonstrates that lidocaine and mepivacaine exhibited a less pronounced influence on chondrogenesis-associated mRNA expression and the course of recovery from the 60-min exposure was better compared with that in the other LA-treated groups.

\section{Discussion}

The results of the present study demonstrated that the viability and apoptotic rate of rADMSCs were decreased and increased, respectively, following a brief exposure to LAs in a concentration- and substance-specific manner in monolayer cell culture experiments during early chondrogenesis. Furthermore, the ADMSC viability and genetic analysis results demonstrated that lidocaine (1\%) and mepivacaine (2\%) appeared to be less toxic to $\mathrm{AADMSC}$ during early chondrogenesis.

Intra-articular administration of amide-type LAs is routinely performed during arthroscopic joint surgery (3). During the last two decades, the use of allogeneic and 
Table I. Primer sequences used for polymerase chain reaction.

Primer sequence (5'-3')

\begin{tabular}{llll} 
Gene & Implication & \multicolumn{1}{c}{ Forward } & Reverse \\
\hline Collagen I & Chondrogenesis & CTGGCCCTAATGGATTCGCT & TCACCCTTAGGTCCCTTGGT \\
Collagen III & Chondrogenesis & TGGAGACTGGGGAAACATGC & CAGAGTCCGTCCACCACTTC \\
Sex-determining region & Chondrogenesis & TCTGGAGACTGCTGAACGAG & CTGCCCATTCTTCACCGACTT \\
Y box 9 & & & \\
GAPDH & Housekeeping gene & ACTGCTGAACGAGAGCGAGAA & CTGCCCATTCTTCACCGACTTC
\end{tabular}
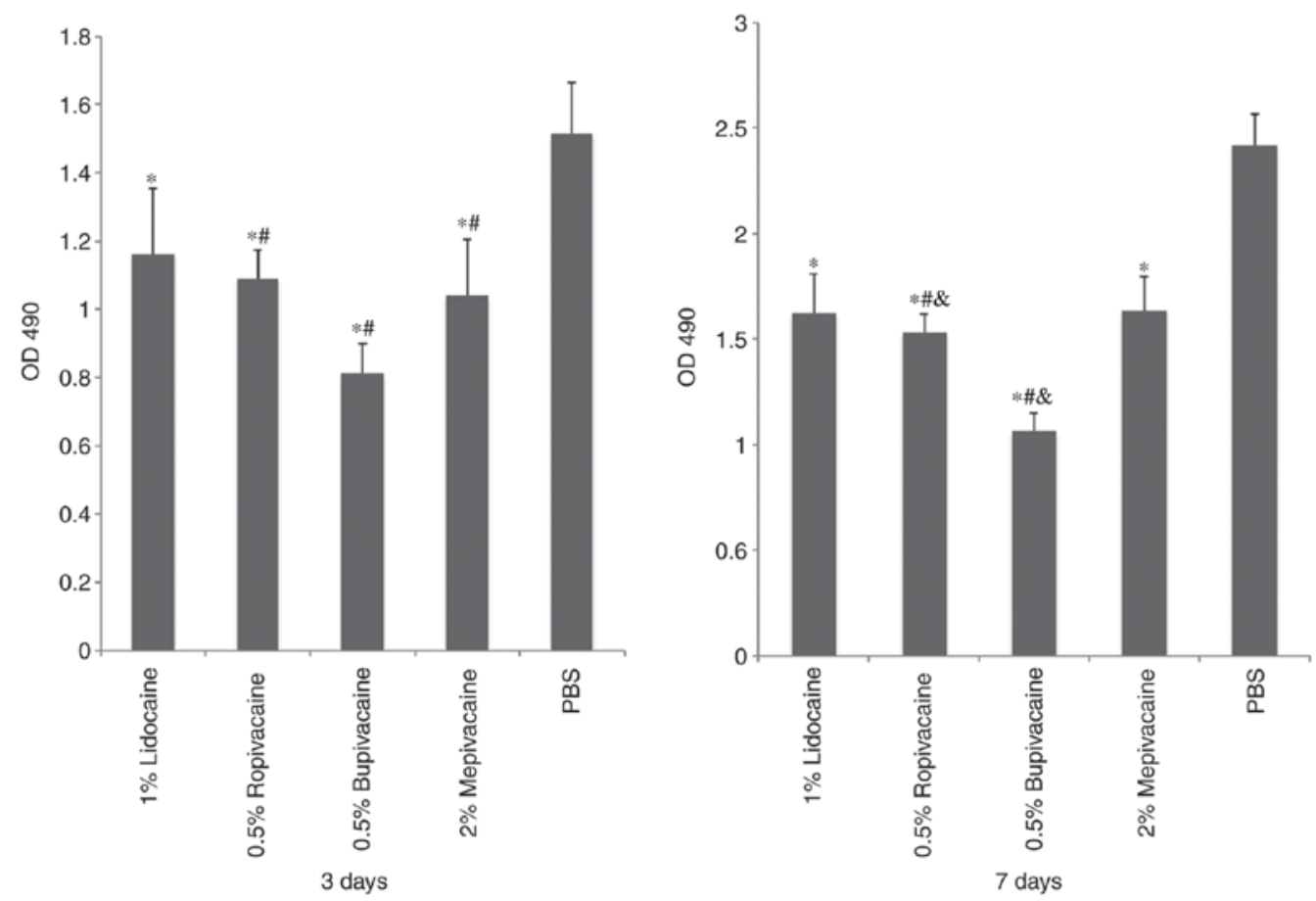

Figure 1. Mitochondrial activity analysis on days 3 and 7 after a 60 -min exposure to LAs determined by an MTS assay. ${ }^{*}<<0.05$ vs. the control group; ${ }^{*} \mathrm{P}<0.05$ vs. the $1 \%$ lidocaine group; ${ }^{\&} \mathrm{P}<0.05$ vs. the $2 \%$ mepivacaine group. LA, local anesthetic; OD, optical density.

autologous human MSCs for tissue repair has become increasingly popular. Stem cell-based methods to treat cartilage defects include marrow-stimulating techniques, including microfracture (14), intra-articular MSC injection (15) and implantation of scaffolding material containing MSCs $(16,17)$. In these instances, the stem cells come into direct contact with intra-articular administered LAs. Different cytotoxic effects of different local anesthetics have been widely reported in patients and animal models. Previous studies have indicated that ropivacaine at its commonly used concentration $(0.5 \%)$ was significantly less toxic than other anesthetics $(0.5 \%$ bupivacaine or $1 \%$ lidocaine) $(6,8)$, and that bupivacaine was more toxic to cartilage cells than ropivacaine and lidocaine (18). However, Keck et al (19) demonstrated that treatment with $2 \%$ lidocaine resulted in a significant reduction in the live cell count compared with that in all other LA-treated groups. The present study demonstrated that lidocaine, bupivacaine, ropivacaine and mepivacaine were cytotoxic to rADMSCs during early chondrogenesis. Lidocaine (1\%) in the present study induced significantly less cytotoxicity compared with ropivacaine, bupivacaine and mepivacaine. Lidocaine and mepivacaine exerted a less pronounced influence on chondrogenesis-associated mRNA expression and the course of recovery from the 60-min exposure was better compared with that in the groups treated with the other LAs. These results may conflict those of previous studies $(8,9)$ as the cytotoxicity effects of LAs on mesenchymal stem cells were different, this may be for the following reasons: Firstly, each of the studies used the LAs at different concentrations; secondly, different methods of analysis, types of MSCs and differentiation conditions were used. Breu et al (9) investigated the cytotoxic potency of LAs on human BMSCs during chondrogenesis. In this experiment, the cells were embedded in varying amounts and structures of cartilage-specific extracellular matrix. In the study by Breu et al (9), it was revealed that bupivacaine, ropivacaine and mepivacaine did not differ in the extent of cytotoxicity induced on MSCs in aggregates during chondrogenesis. It is not unusual that different concentrations and MSC conditions 


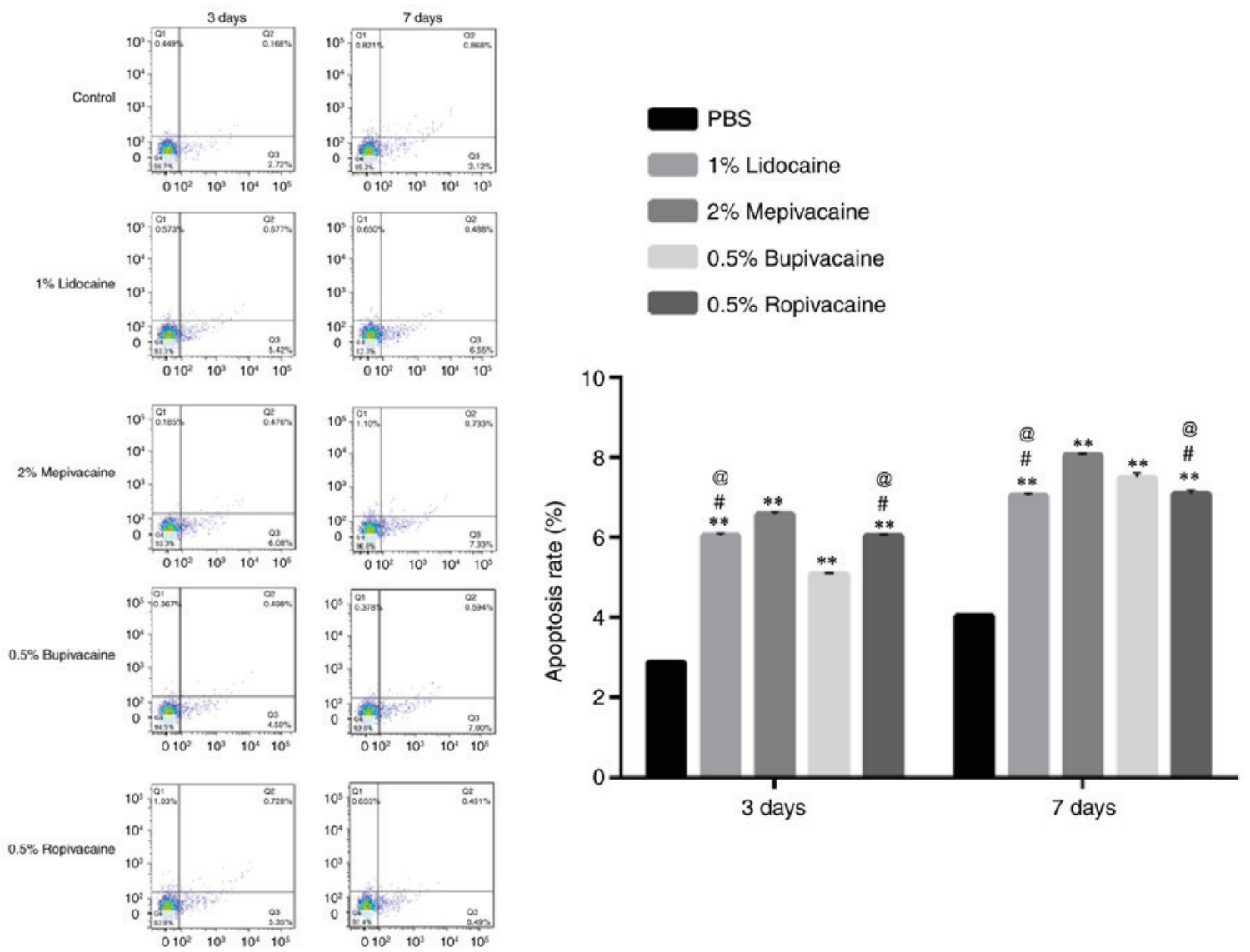

Figure 2. Apoptosis analysis after a 60 -min exposure to LAs followed by 3 or 7 days of culture. ${ }^{* *} \mathrm{P}<0.01$ vs. the control group; ${ }^{*} \mathrm{P}<0.05$ vs. the $2 \%$ mepivacaine group; ${ }^{\circledR} \mathrm{P}<0.05$ vs. the $0.5 \%$ bupivacaine group. LA, local anesthetic.
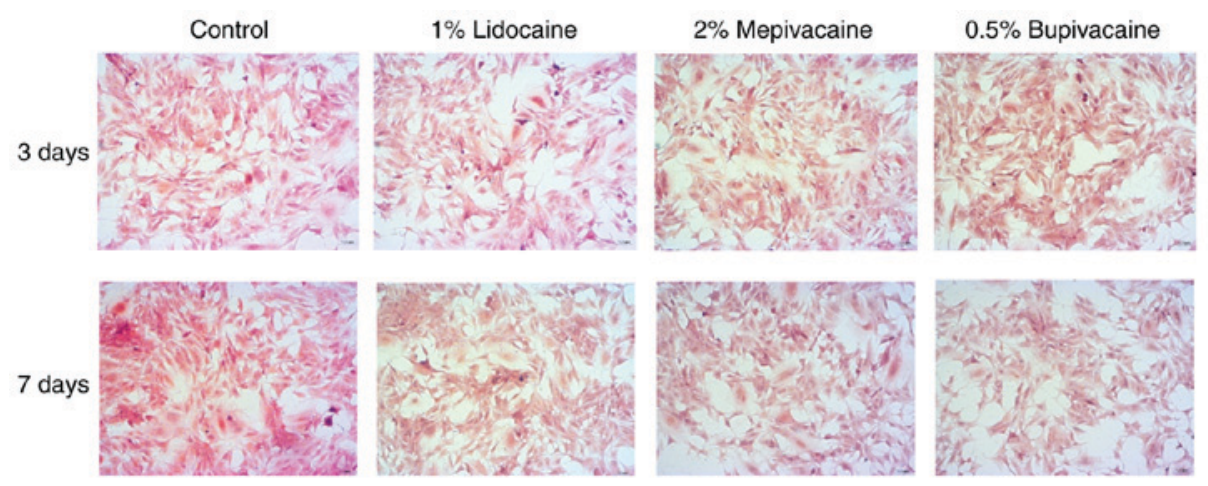

$0.5 \%$ Ropivacaine
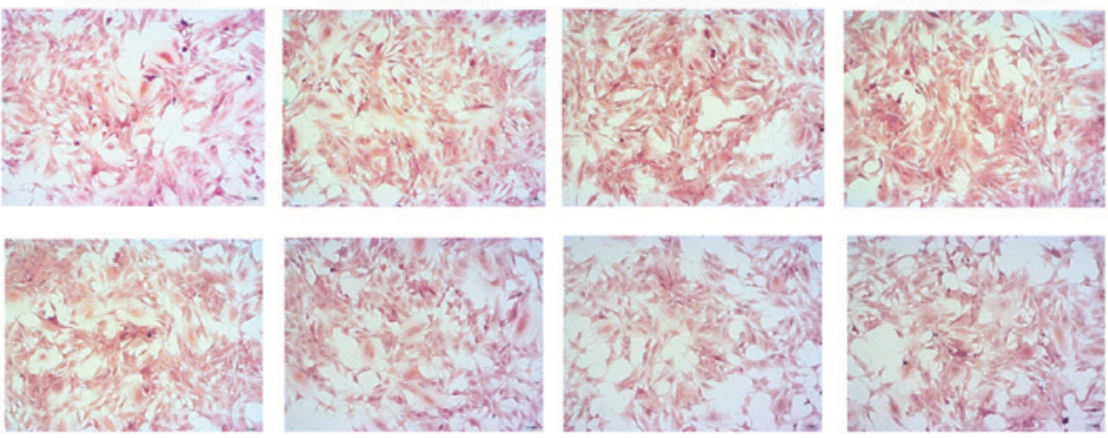

Figure 3. Effect of exposure to LAs on morphological alterations of chondrogenic differentiation (Safranine Fast Green double staining; magnification, $\mathrm{x} 400$ ). LA treatment resulted in less mature fibrocartilage compared with PBS on days 3 and 7 following a 60-min exposure. In the $1 \%$ lidocaine treatment group, adipose-derived mesenchymal stem cell pellets resembled more mature fibrocartilage compared with those in the other LA groups, as demonstrated by staining. LA, local anesthetic.

(e.g. cell types and direction of differentiation) lead to different results. In clinical practice, $1 \%$ lidocaine, $0.5 \%$ bupivacaine, $0.5 \%$ ropivacaine and $2 \%$ mepivacaine is utilized at our institution. To mimic the clinical situation, the cytotoxicity of these anesthetics was compared at these concentrations. The present study assessed the cytotoxic effects of LAs with equivalent anesthetic potencies. To model the conditions of early chondrogenesis, the rADMSCs were allowed to differentiate for up to 3 days in chondrogenic differentiation media and were subsequently exposed to LAs for $60 \mathrm{~min}$. Therefore, the experimental conditions used in the present study are closest to the ones used in clinical practice.

All local anesthetics used in the present study are members of the amide family. The cytotoxicity of LAs on different cell types, including neurons, has been addressed by several studies $(20,21)$. Three major mechanisms have been hypothesized to explain the cytotoxic effects of LAs on stem cells. First, the toxic actions of local anesthetics have been demonstrated to be associated with their lipophilic properties $(22,23)$. Bupivacaine and ropivacaine are highly lipophilic 

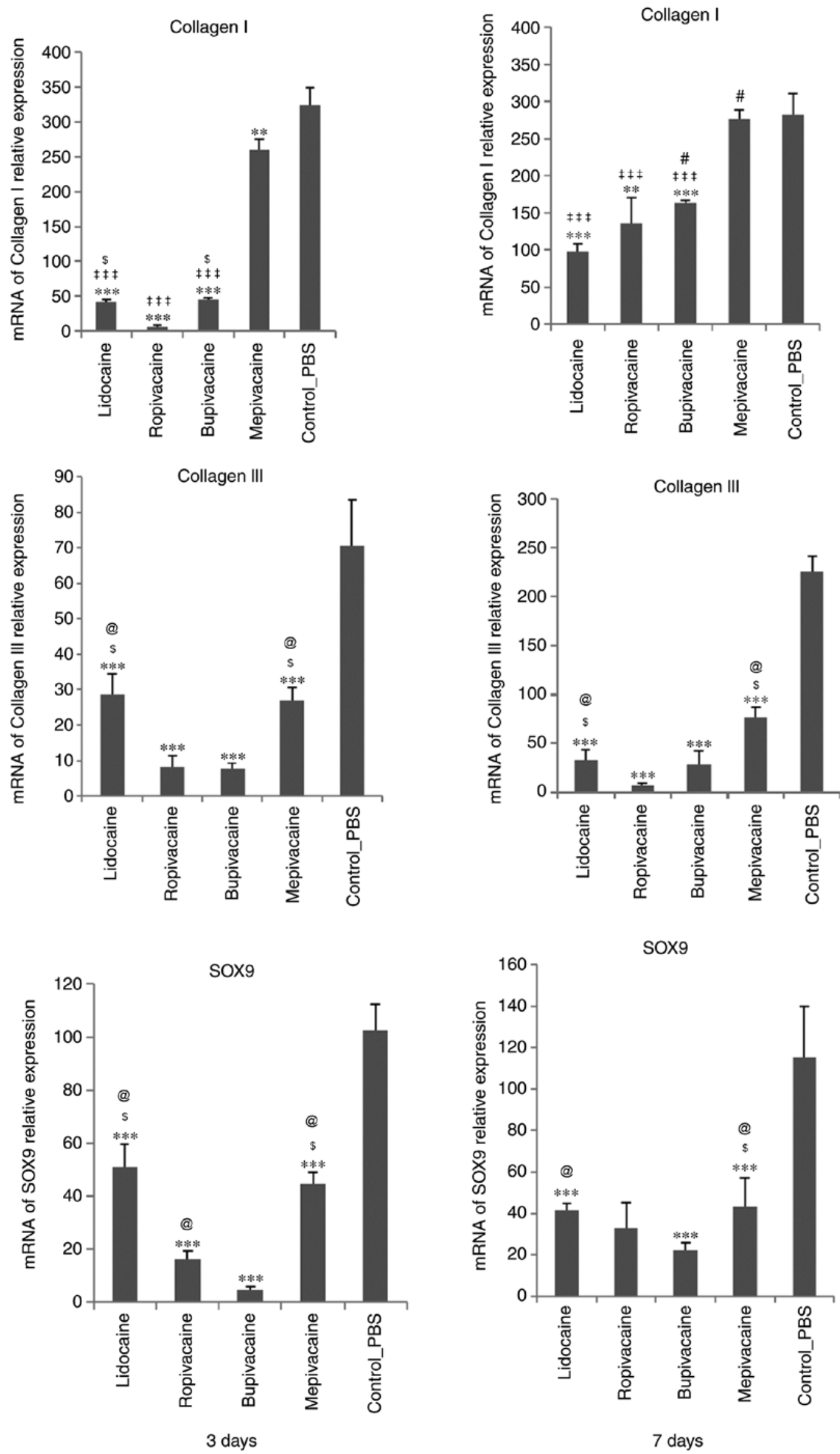

Figure 4. Chondrogenesis-associated gene expression analysis of rabbit adipose-derived mesenchymal stem cells in response to a 60-min exposure to LAs determined by reverse transcription-quantitative polymerase chain reaction analysis at days 3 and 7 after the exposure. ${ }^{* * *} \mathrm{P}<0.01,{ }^{* * * *} \mathrm{P}<0.001 \mathrm{vs}$. control group; ${ }^{\#} \mathrm{P}<0.05$ vs. the $0.5 \%$ lidocaine group; ${ }^{\$} \mathrm{P}<0.05$ vs. the $0.5 \%$ ropivacaine groups; ${ }^{\circledR} \mathrm{P}<0.05$ vs. the $0.5 \%$ bupivacaine; ${ }^{\#+} \mathrm{P}<0.001$ vs. $2 \%$ mepivacaine group. $\mathrm{LA}$, local anesthetic; SOX9, sex-determining region Y box 9. 
molecules, whereas lidocaine is only slightly lipophilic (24). Furthermore, MSCs are sensitive to LAs that may inhibit cell-to-cell communication, which is critical in proliferation and migration, and inhibition of mitochondrial respiration with subsequent depletion of cellular ATP and formation of reactive oxygen species may occur $(25,26)$. LAs may also affect inflammatory processes. Bupivacaine was reported to induce nitric oxide synthase-2 activity (27). This suggested that bupivacaine toxicity may be in part attributable to the production of nitric oxide during ongoing inflammation. In terms of cell viability and apoptosis, $1 \%$ lidocaine exhibited less cytotoxicity on ADMSCs than the other LAs used in the present study. This result is similar to those of previous studies. Shoshani et al (28) investigated the effect of lidocaine on the viability of injected adipose tissue in nude mice. A local anesthetic solution, consisting of lidocaine and epinephrine, did not alter the acceptance of fat grafts, and had no influence on the viability of adipocytes. However, in terms of gene expression, $2 \%$ mepivacaine appeared to have a low impact on the expression of cartilage differentiation-associated genes. These results imply that different local anesthetics exert toxic effects on ADMSCs during cartilage differentiation via different mechanisms. Therefore, further in vitro and in vivo studies using equipotent LAs are required to confirm the mechanisms of cytotoxicity on ADMSCs during cartilage differentiation.

One limitation of the present study is the fact that the experiments were performed in vitro. The drug concentration was constant over the 60-min exposure time. However, drug concentrations slowly decrease over time in clinical practice. Therefore, further experiments are required to assess the in vivo cytotoxic effects of LAs on ADMSCs.

In conclusion, the present study demonstrated that several common amide-type LAs are cytotoxic to rADMSCs during early chondrogenesis in a drug type-dependent manner. Furthermore, $1 \%$ lidocaine exerted relatively lower cytotoxic effects on ADMSCs, and 2\% mepivacaine and $1 \%$ lidocaine appeared to exhibit a less pronounced effect on cartilage differentiation-associated gene expression. In the light of the results of the present study, it is of critical importance that clinicians are cautious when selecting an amide-type LA to be used during rADMSC therapy in future, in order to avoid compromising the integrity and potency of cell-based therapies.

\section{Acknowledgements}

The authors of the present study give thanks to Dr. Jinjin Ben (Pathology Department, Nanjing Medical University, Nanjing, China) for their technical assistance.

\section{Funding}

This study was supported by the Natural Science Foundation of Zhejiang Province, China (2016-2018; grant no. LY16H170001).

\section{Availability of data and materials}

The datasets used and/or analyzed during the current study are available from the corresponding author on reasonable request.

\section{Authors' contributions}

TW and JL designed and directed the experiment. ZS, HS and YL performed the experiments. ZS performed the statistical analysis. TW and JL wrote the manuscript. ZS and HS reviewed and edited the manuscript. The final version of the manuscript has been read and approved by all authors, and each author believes that the manuscript represents honest work.

\section{Ethical approval and consent to participate}

The experimental protocol was approved by the Animal Ethics Committee of Zhejiang University Affiliated with SRRSH (Hangzhou, China).

\section{Patient consent for publication}

Not applicable.

\section{Competing interests}

All authors declare that they have no competing interests.

\section{References}

1. Vos T, Flaxman AD, Naghavi M, Lozano R, Michaud C, Ezzati M, Shibuya K, Salomon JA, Abdalla S, Aboyans V, et al: Years lived with disability (YLDs) for 1160 sequelae of 289 diseases and injuries 1990-2010: A systematic analysis for the Global Burden of Disease Study 2010. Lancet 380: 2163-2196, 2010.

2. Wu T, Song HX, Dong Y and Li JH: Cell-based therapies for lumbar discogenic low back Pain: Systematic review and single-arm meta-analysis. Spine (Phila Pa 1976) 43: 49-57, 2018.

3. Møiniche S, Mikkelsen S, Wetterslev J and Dahl JB: A systematic review of intra-articular local anesthesia for postoperative pain relief after arthroscopic knee surgery. Reg Anesth Pain Med 24: 430-437, 1999.

4. Ballieul RJ, Jacobs TF, Herregods S, Van Sint Jan P, Wyler B, Vereecke H, Almqvist F and Herregods L: The peri-operative use of intra-articular local anesthetics: A review. Acta Anaesthesiol Belg 60: 101-108, 2009.

5. Chu CR, Izzo NJ, Coyle CH, Papas NE and Logar A: The in vitro effects of bupivacaine on articular chondrocytes. J Bone Joint Surg Br 90: 814-820, 2008.

6. Dregalla RC, Lyons NF, Reischling PD and Centeno CJ: Amide-type local anesthetics and human mesenchymal stem cells: Clinical implications for stem cell therapy. Stem Cells Transl Med 3: 365-374, 2014.

7. Lucchinetti E, Awad AE, Rahman M, Feng J, Lou PH, Zhang L, Ionescu L, Lemieux H, Thébaud B and Zaugg M: Antiproliferative effects of local anesthetics on mesenchymal stem cells: Potential implications for tumor spreading and wound healing. Anesthesiology 116: 841-856, 2012.

8. Breu A, Eck1 S, Zink W, Kujat R and Angele P: Cytotoxicity of local anesthetics on human mesenchymal stem cells in vitro. Arthroscopy 29: 1676-1684, 2013.

9. Breu A, Scheidhammer I, Kujat R, Graf B and Angele P: Local anesthetic cytotoxicity on human mesenchymal stem cells during chondrogenic differentiation. Knee Surg Sports Traumatol Arthrosc 23: 937-945, 2015

10. Zhu Y, Liu T, Song K, Fan X, Ma X and Cui Z: Adipose-derived stem cell: A better stem cell than BMSC. Cell Biochem Funct. 26: 664-675, 2008.

11. Romeo R, Carnovale C, Giofrè SV, Monciino G, Chiacchio MA, Sanfilippo C and Macchi B: Enantiomerically pure phosphonated carbocyclic 2'-oxa-3'-azanucleosides: Synthesis and biological evaluation. Molecules 19: 14406-14016, 2014.

12. Pascual Garrido C, Hakimiyan AA, Rappoport L, Oegema TR, Wimmer MA and Chubinskaya S: Anti-apoptotic treatments prevent cartilage degradation after acute trauma to human ankle cartilage. Osteoarthritis Cartilage 17: 1244-1251, 2009. 
13. Livak KJ and Schmittgen TD: Analysis of relative gene expression data using real-time quantitative PCR and the 2(-Delta Delta C (T)) method. Methods 25: 402-408, 2001.

14. Asik M, Ciftci F, Sen C, Erdil M and Atalar A: The microfracture technique for the treatment of full-thickness articular cartilage lesions of the knee: Midterm results. Arthroscopy 24: 1214-1220, 2008.

15. Xia P, Wang X, Lin Q and Li X: Efficacy of mesenchymal stem cells injection for the management of knee osteoarthritis: A systematic review and meta-analysis. Int Orthop 39: 2363-2372, 2015.

16. Løken S1, Jakobsen RB, Arøen A, Heir S, Shahdadfar A Brinchmann JE, Engebretsen L and Reinholt FP: Bone marrow mesenchymal stem cells in a hyaluronan scaffold for treatment of an osteochondral defect in a rabbit model. Knee Surg Sports Traumatol Arthrosc 16: 896-903, 2008.

17. Haleem AM, Singergy AA, Sabry D, Atta HM, Rashed LA, Chu CR, El Shewy MT, Azzam A and Abdel Aziz MT: The clinical use of human culture-expanded autologous bone marrow mesenchymal stem cells transplanted on platelet-rich fibrin glue in the treatment of articular cartilage defects: A pilot study and preliminary results. Cartilage 1: 253-261, 2010.

18. Piper SL, Kramer JD, Kim HT and Feeley BT: Effects of local anesthetics on articular cartilage. Am J Sports Med 39: 2245-2253, 2011

19. Keck M,Zeyda M, Gollinger K, Burjak S, Kamolz LP, Frey M and Stulnig TM: Local anesthetics have a major impact on viability of pre-adipocytes and their differentiation into adipocytes. Plast Reconstr Surg 126: 1500-1505, 2010.

20. Chang YS, Tseng SY, Tseng SH and Wu CL: Cytotoxicity of lidocaine or bupivacaine on corneal endothelial cells in a rabbit model. Cornea 25: 590-596, 2006.

21. Pollock JE: Neurotoxicity of intrathecal local anaesthetics and transient neurological symptoms. Best Pract Res Clin Anaesthesiol 17: 471-484, 2003.
22. Punke MA and Friederich P: Lipophilic and stereospecific interactions of amino-amide local anesthetics with human Kv1.1 channels. Anesthesiology 109: 895-904, 2008.

23. Werdehausen R, Fazeli S, Braun S, Hermanns H, Essmann F, Hollmann MW, Bauer I and Stevens MF: Apoptosis induction by different local anaesthetics in a neuroblastoma cell line. Br J Anaesth 103: 711-718, 2009.

24. Grouselle M, Tueux O, Dabadie P, Georgescaud D and Mazat JP: Effect of local anaesthetics on mitochondrial membrane potential in living cells. Biochem J 271: 269-272, 1990.

25. Fu X, Han B, Cai S, Lei Y, Sun T and Sheng Z: Migration of bone marrow-derived cells induced by tumor necrosis factor-alpha and its possible role in wound healing. Wound Repair Regen 17: 185-191, 2009.

26. Tanaka Y, Maruo A, Fujii K, Nomi M, Nakamura T, Eto S and Minami Y: Intercellular adhesion molecule 1 discriminates functionally different populations of human osteoblasts: Characteristic involvement of cell cycle regulators. J Bone Miner Res 15: 1912-1923, 2000.

27. Feinstein DL, Murphy P, Sharp A, Galea E, Gavrilyuk V and Weinberg G: Local anesthetics potentiate nitric oxide synthase type 2 expression in rat glial cells. J Neurosurg Anesthesiol 13: 99-105, 2001

28. Shoshani O, Berger J, Fodor L, Ramon Y, Shupak A, Kehat I, Gilhar A and Ullmann Y: The effect of lidocaine and adrenaline on the viability of injected adipose tissue: An experimental study in nude mice. J Drugs Dermatol 44: 311-316, 2005.

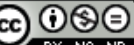

This work is licensed under a Creative Commons Attribution-NonCommercial-NoDerivatives 4.0 International (CC BY-NC-ND 4.0) License. 